\title{
The star formation history of the Fornax dSph galaxy using the new synthetic color-magnitude diagram code IAC-STAR
}

\author{
Carme Gallart, ${ }^{1}$ Antonio Aparicio, ${ }^{1}$ R. Zinn, ${ }^{2}$ \\ R. Buonanno, ${ }^{3}$ E. Hardy ${ }^{4}$ and G. Marconi ${ }^{5}$ \\ ${ }^{1}$ Instituto de Astrofísica de Canarias, 38200 La Laguna, Tenerife, Spain \\ email: carme@iac.es, antapaj@iac.es \\ ${ }^{2}$ Dep. of Astronomy, Yale University, P.O. Box 208101, New Haven, CT 06520-8101, USA, \\ email: zinn@astro.yale.edu \\ ${ }^{3}$ Università di Roma Tor Vergata, via della Ricerca Scientifica 1, 00133 Rome, Italy, \\ email: buonanno@mporzio.astro.it \\ ${ }^{4}$ NRAO, Santiago de Chile, email: ehardy@nrao.edu \\ ${ }^{5}$ ESO, Alonso de Cordova 3107, Vitacura, Santiago de Chile, email: gmarconi@eso.org
}

\begin{abstract}
We present VLT-FORS1 color-magnitude diagrams (CMDs) reaching the oldest main-sequence turnoffs for three fields at two galactocentric distances in the Fornax dSph galaxy. We derive the star formation history ( $\mathrm{SFH}$ ) for each field through comparison with synthetic CMDs and show that the main epoch of star formation has extended to more recent epochs toward the center of the galaxy. A small burst of star formation about 2 Gyr ago is a robust feature in the SFH of all fields. It might be related to the shell structure found in Fornax by Coleman et al. (2004), which in turn has been interpreted as the relic of a merger event that occurred in Fornax about 2 Gyr ago.
\end{abstract}

\section{Introduction}

The Fornax dwarf spheroidal (dSph) galaxy is - in competition with the Sagittarius dwarf - the most luminous and massive dSph satellite in the Milky Way. Both contain their own globular cluster system. In addition, except for Leo I and Leo II, Fornax is the most distant satellite of the Milky Way. Early hints that Fornax contained a substantial population of intermediate-age stars were provided by the discovery of a large number of C stars by Aaronson \& Mould (1980). The first evidence of bright main-sequence stars was provided by Buonanno et al. (1985). The intermediate-age and young (down to $100 \mathrm{Myr}$ ) population of Fornax, and its stellar population gradients were shown clearly for the first time by Stetson, Hesser, \& Smecker-Hane (1998) with a color-magnitude diagram (CMD) of 100000 stars on a $\frac{1}{3} \mathrm{deg}^{2}$ field. A deeper HST-WFPC2 CMD reaching the oldest main-sequence turnoffs in a small field near the center of the galaxy was presented by Buonanno et al. (1999). This study indicated that star formation began in the Fornax field $\simeq 12$ Gyr ago and continued to at least 0.5 Gyr ago. Apparently separated subgiant branches provided tantalizing evidence that star formation occurred in discrete bursts.

In this paper, we present VLT-FORS1 CMDs as deep as the HST one obtained by Buonanno et al. (1999) but covering a much larger area, and sampling the Fornax stellar populations at two galactocentric radius. Preliminary results on the detailed star formation history $(\mathrm{SFH})$ in these fields, obtained through comparison with synthetic CMDs, are discussed. 


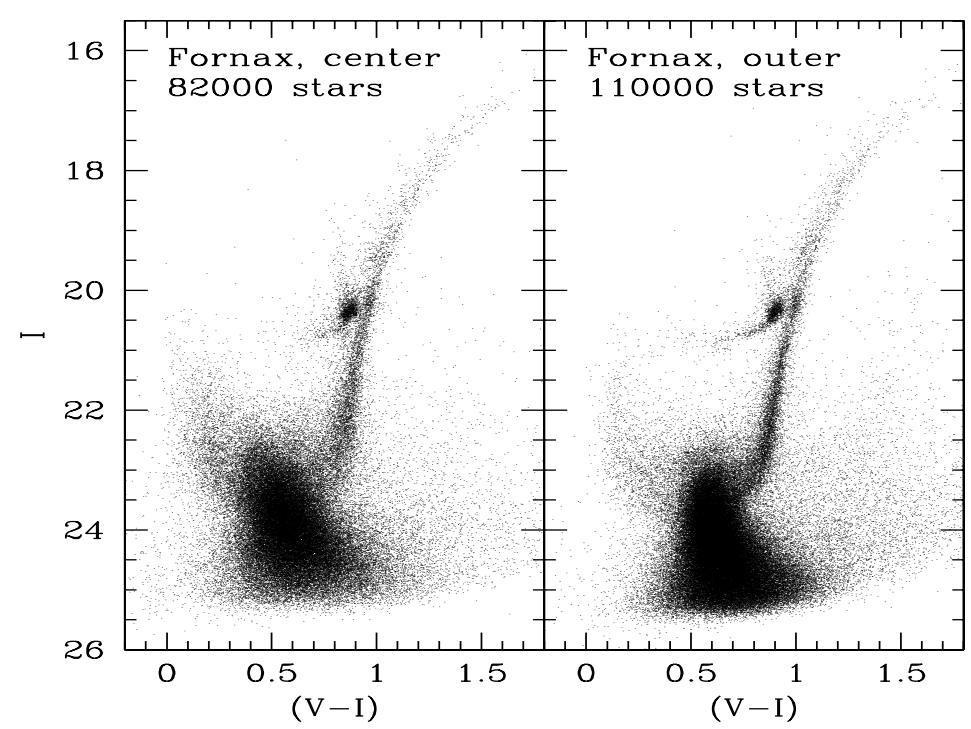

Figure 1. VLT CMDs of Fornax for the central and the outer fields discussed in the text.

\section{The Data}

We obtained old main-sequence turnoff photometry for three fields at two galactocentric distances in Fornax using FORS1 at the VLT. The observations were performed in service mode in July 2000. The seeing was typically $0.6^{\prime \prime}$, but in some of the frames, with exposure times as long as $750 \mathrm{~s}$, the measured seeing was as good as $0.4^{\prime \prime}$. In the central field containing globular cluster Fornax\#4, a total of $3650 \mathrm{~s}$ in $V$ and $4700 \mathrm{~s}$ in $I$ were accumulated. The other two fields were situated at about 11 arcmin north of the center of the galaxy, and total integration times were $1850 \mathrm{~s}$ in $V$ and $4600 \mathrm{~s}$ in $I$. The locations of these off-center fields were chosen taking into account the change in surface brightness across Fornax and selecting fields that would contain similar numbers of stars in the central field and in the other two fields combined.

Figure 1 shows the corresponding CMDs. Note that their depth is similar to that of the HST-WFPC2 CMD by Buonanno et al. (1999), but that the number of stars is much larger, thus providing a better representation of the $\mathrm{SFH}$ that is less affected by small number statistics.

\section{The synthetic CMD code: IAC-STAR}

We are performing the analysis of the Fornax CMDs in terms of the SFH and its gradients using the new codes IAC-STAR (available on the Web at http://iac-star.iac.es; Aparicio \& Gallart 2004) and IAC-POP (Hidalgo \& Aparicio 2005), using a similar method as described in Gallart et al. (1999).

IAC-STAR allows the computation of synthetic CMDs for any combination of the star formation rate, $\operatorname{SFR}(t)$, chemical enrichment, $Z(t)$, initial mass function (IMF), and frequency and mass distribution of binary stars. Composite stellar populations are calculated on a star-by-star basis, by computing the luminosity, effective temperature, and gravity of each star by direct interpolation in the metallicity and age grid of a library of stellar evolution tracks. Visual and infrared magnitudes are also provided for each star after applying bolometric corrections. The version currently available offers the 
choice of the stellar evolution libraries of Bertelli et al. (1994), Girardi et al. (2000), and Pietrinferni et al. (2004), as well as various bolometric corrections libraries.

To compare model and observations, the synthetic stars are distributed in an array of "partial" models containing stars within small intervals of age and metallicity, and a set of boxes is defined in the CMD. An array, $M_{i}^{j}$, containing the number of stars from partial model $S_{i}$ populating box $j$ is computed. The same operation is made in the observational $\mathrm{CMD}$, producing a vector $O^{j}$ containing the number of observed stars in box $j$. With this information, the distribution of stars in the defined boxes can be calculated for any SFH as a linear combination of the $M_{i}^{j}: M^{j}=A \sum_{i} \alpha_{i} M_{i}^{j}$, where $A$ is a scaling constant. The $\mathrm{SFH}$ best matching the distribution, $O^{j}$, of the observational CMD can be found using a merit function such as $\chi^{2}$ or, better, $\chi_{\gamma}^{2}$ (Mighell 1999), which provides the best solution and a test of its goodness. Finally, the corresponding SFH can be written as $S=A \sum_{i} \alpha_{i} S_{i}$. The best-fitting SFH has been calculated using the code IAC-POP, which uses a genetic algorithm (Charbonneau 1995) to optimize the minimization of $\chi_{\gamma}^{2}$.

\section{The star formation history and its gradients}

In this section, we present preliminary results of our derivation of the Fornax SFH. The grid of synthetic CMDs used combined the following parameters and functions:

i) Ten $Z(t)$ producing a color distribution in the model CMDs compatible with the observed one; ii) four values of the binary fraction, $f=0.1,0.3,0.6,0.9$, assuming a minimum mass ratio $q=0.6$; and iii) three parameterizations of the IMF, as in Gallart et al. (1999), with a common slope $m^{-1.3}$ for $0.08 \leqslant m \leqslant 0.5$, as in Kroupa, Tout, \& Gilmore (1993) for the disk of the Milky Way, and three different slopes for the upper mass interval bracketing those of Kroupa et al.

The Bertelli et al. (1994; Padova hereinafter) and Pietrinferni et al. (2004; Teramo) stellar evolution models were used to create synthetic CMDs with the grid of ingredients listed above. The comparison of these synthetic CMDs with the observed CMD, after the observational errors were simulated in them following the procedure described in Gallart et al. (1999), allowed us to identify a number of $Z(t)$ for which we can find an $\operatorname{SFR}(t)$ that successfully reproduces the number of stars across the observed CMD. It was not possible to discriminate between the different parameterizations of the IMF. While strictly speaking no unique solution could be found, the accepted solutions define a narrow range of $Z(t)$ and $\operatorname{SFR}(t)$ combinations. The $Z(t)$ range is compatible with the age-metallicity relation derived by Pont et al. (2004) from CaII triplet spectroscopy. In Figure 2, examples of these solutions are displayed.

The main difference between the $\operatorname{SFR}(t)$ in the central and outer fields, which appears independently of the stellar evolution model set used, is that the main epoch of star formation ends about 5 Gyr ago in the outer field, while it extends up to 3 Gyr ago in the central field. Low-intensity star formation, more prominent in the center of Fornax, has continued till just a few hundred Myr ago. In the case of the Padova models, the existence of a small burst about 1.5 Gyr ago is a robust and ubiquitous feature that appears in basically all accepted solutions. This feature disappears, or rather, gets shifted to older ages, in the solutions with the Teramo models. This is a consequence of the known differences between these two model sets, as discussed in Gallart, Zoccali, \& Aparicio (2005). In particular, at intermediate ages, the Teramo models assign an older age to stars in the same position of the CMD than the Padova models. For example, a $Z=0.001,2$ Gyr old Teramo isochrone matches almost perfectly a 1 Gyr old Padova isochrone. The age of the young burst of star formation, as inferred with the Padova models, is in very good agreement with the age of the stellar population that dominates the shell structure 


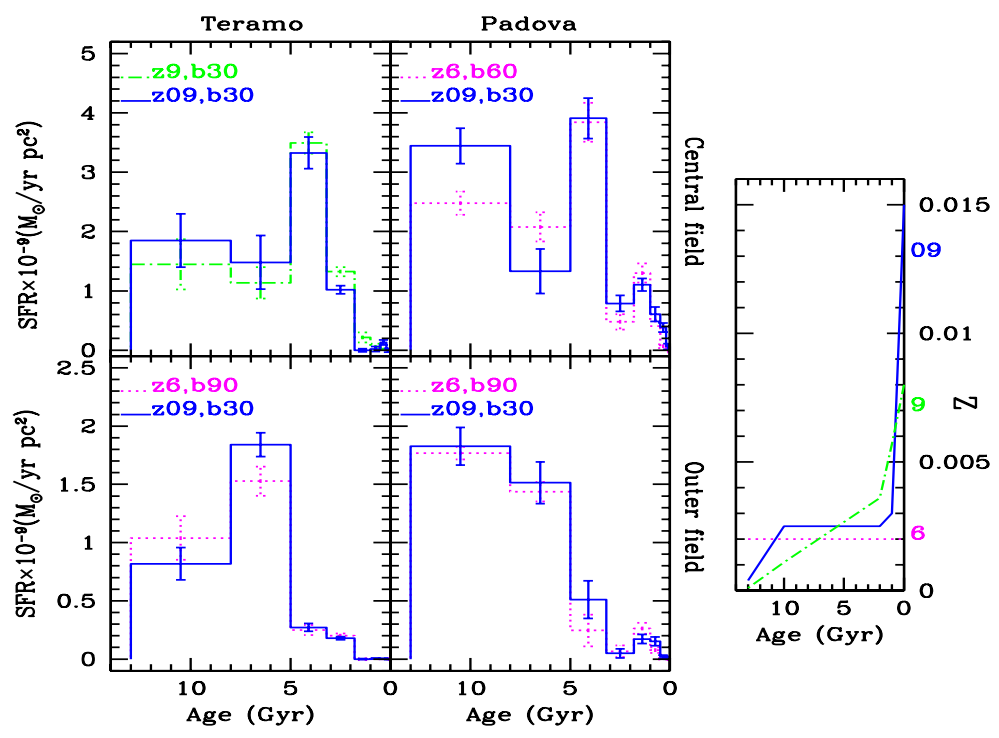

Figure 2. SFHs derived for the two Fornax fields (see text for details), for both the Teramo and Padova models. The labels in each panel indicate the assumed metallicity law (the three possibilities are displayed on the right panel, with their corresponding code indicated), and the percentage of binary stars.

found in Fornax by Coleman et al., (2004), which was in fact dated using the same models. These authors interpret this shell structure possibly as the remnant of a merger with Fornax of a smaller, gas-rich system, about 2 Gyr ago. Since we find the increased SFR at approximately the same age in all three fields sampled, this may indicate that the stars incorporated into Fornax by this smaller system are not confined to the shell structure but have mixed substantially over a large volume of the galaxy.

\section{Acknowledgements}

This research is part of a Joint Project between Universidad de Chile and Yale University, partially funded by the Fundación Andes. CG acknowledges partial support from the Spanish Ministry of Science and Technology (Plan Nacional de Investigación Científica, Desarrollo e Investigación Tecnológica, AYA2004-06343) and from the European Structural Funds.

\section{References}

Aaronson, M. \& Mould, J. 1980, ApJ 240, 804

Aparicio, A. \& Gallart, C. 2004, AJ 128, 1465

Bertelli, G., Bressan, A., Chiosi, C., Fagotto, F. \& Nasi, E. 1994, A\&AS 106, 275

Buonanno, R., Corsi, C.E., Fusi Pecci, F., Hardy, E. \& Zinn, R. 1985, A\&JA 152, 65

Buonanno, R., Corsi, C.E., Castellani, M., Marconi, G., Fusi Pecci, F. \& Zinn, R. 1999, AJ 118, 1671

Charbonneau, P. 1995, ApJS 101, 309

Coleman, M., Da Costa, G.S., Bland-Hawthorn, J., Martínez-Delgado, D., Freeman, K.C. \& Malin, D. 2004, AJ 127, 832

Gallart, C., Freedman, W.L., Aparicio, A., Bertelli, G. \& Chiosi, C. 1999, AJ 118, 2245

Gallart, C. Zoccali, M. \& Aparicio, A. 2005, ARAA 43, in press

Girardi, L., Bressan, A., Bertelli, G. \& Chiosi, C. 2000. A\&AS 141, 371 
Hidalgo, S. \& Aparicio, A. 2005, in preparation

Kroupa, P., Tout, C.A. \& Gilmore, G. 1993, MNRAS 262, 545

Mighell, K.J. 1999. ApJ 518, 380

Pietrinferni, A., Cassisi, S., Salaris, M. \& Castelli, F. 2004, ApJ 612, 168

Stetson, P.B., Hesser, J.E. \& Smecker-Hane, T.A. 1998, PASP 110, 533

\section{Discussion}

MATEO: Do you see different spatial distributions as a function of age? I know the field is small, but the young clump may inject stars in a highly asymmetric manner.

GALlART: It is a good idea. But I haven't actually analyzed this yet. However, note that the "excess" young population seems to be found everywhere we look, even in the off-center field, which is situated opposite to the shell structure with respect to the center of the galaxy.

DA Costa: Can you briefly summarize the differences between the Padova and Teramo evolutionary models? A factor of two difference in age at 1 Gyr is somewhat embarrassing.

GALlART: The Teramo models have updated most physics input to very recent determinations (equation of state, nuclear reactions, etc.). In this age range the overshooting treatment is also very relevant. It is very difficult to isolate which are the key ingredients that produce this remarkable difference. Pietrinferni et al. (2004) briefly discuss the possible origin of the discrepancy and conclude that only a fraction of it is related to differences in the overshooting treatment, the remainder having to be ascribed to the different input physics. But they didn't isolate the key ingredient. I think it would be very important, in any case, to fine-tune the overshooting treatment, through comparison with observations, specifically for each stellar evolution library.

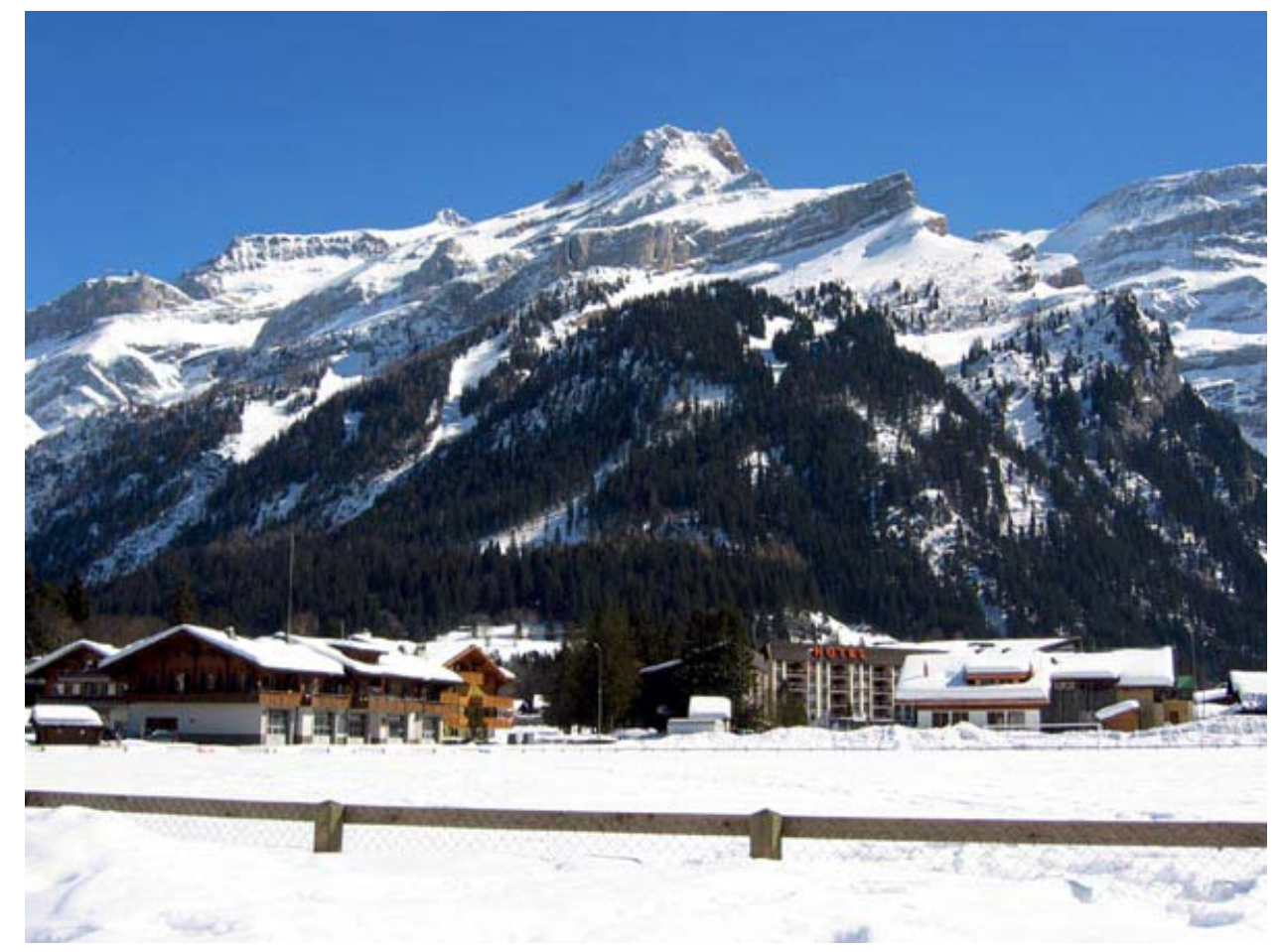

